\title{
Fever and Dehydration as the First COVID-19 Presentation in a 2-Months Old Boy in Northeast of Iran
}

\author{
Mahsa Besharat (iD ${ }^{1,}$ " and Pooya Bahari Khorram ${ }^{2}$ \\ ${ }^{1}$ Pediatrician, Infectious Diseases Research Center, Golestan University of Medical Sciences, Gorgan, Iran \\ ${ }^{2}$ Radiologist, Esfarayen University of Medical Sciences, Esfarayen, Iran \\ "Corresponding author: Infectious Diseases Research Center, Golestan University of Medical Sciences, Gorgan, Iran. Email: mahsabe.mb@gmail.com \\ Received 2020 July 08; Revised 2020 August 02; Accepted 2020 August 05.
}

\begin{abstract}
Introduction: After the worldwide pandemic of coronavirus 19 disease (COVID-19), pediatric involvement has been seen as case reports with various initial symptoms.

Case Presentation: Here, we report a 2-months old boy with severe dehydration, fever, and electrolyte imbalance with lung involvement compatible with COVID-19 in the Northeast of Iran (North Khorasan).

Conclusions: Pediatric case reports in COVID-19 are limited, and it seems that the disease has a milder course compared to the adult patients, but the range of the clinical features is wide.
\end{abstract}

Keywords: Electrolyte Imbalance, COVID-19, Iran

\section{Introduction}

Since the worldwide pandemic of novel coronavirus 19 disease (COVID-19), different clinical presentations have been reported in children with various disease courses. At first, the main presentations were respiratory, and it seemed that this virus could not affect children under one year old. However, different case reports showed that the symptoms and clinical courses in the pediatric population could range from respiratory involvement to gastrointestinal symptoms and even Kawasaki disease that has been reported lately $(1,2)$. Here, we report a 2-months old boy with dehydration and electrolytes imbalance referring to our hospital with lung involvement in his CT-scan.

\section{Case Presentation}

On 3rd of March 2020, a 2-months old boy referred to our hospital with severe dehydration and fever from 4 days ago. He had received cefixime suspension and oral ondansetron and oral acetaminophen for fever in the outpatient clinic with no obvious improvements in the clinical course. His mother had respiratory symptoms such as cough and mild chest pain, and there was a positive history of contact with a person who had returned from one of the epidemic areas in Iran. His first physical examination revealed severe dehydration. He had a $38^{\circ} \mathrm{C}$ fever, and his heart rate was 180 beats/min, respiratory rate was $40 / \mathrm{min}$, and blood pressure of $80 / 50 \mathrm{mmHg}$. His first $\mathrm{O}_{2}$ saturation detected by finger pulse oximetry was $92 \%$.

He was admitted to the pediatric ward in Northeast of Iran (North Khorasan), and we started intravenous (IV) fluid resuscitation with $20 \mathrm{cc} / \mathrm{kg}$ normal saline and then IV dextrose 5\% and half saline with 40 meq/L KCl 15\% (calculating based upon maintenance and dehydration). Blood tests were carried out, and the initial therapy was based on the diagnosis of possible sepsis with IV ampicillin and cefotaxime. He had no respiratory distress, but his $\mathrm{O}_{2}$ sat was constantly lower than $90 \%$, so we started free $\mathrm{O}_{2}$ flow 5 - 6 lit/min. His first Chest X-Ray was normal.

His laboratory test results were as follows:

A complete sepsis workup was done for him. The laboratory results revealed: White blood cell $(\mathrm{WBC})=\mathbf{2 5 0 0 0}$ (with 50\% Neutrophil), a positive C-reactive protein (CRP) and erythrocyte sedimentation rate (ESR) was 25 in one hour. Urine analysis and culture were performed that were negative. The lumbar puncture was not remarkable. His blood sodium level was $168 \mathrm{mEq} / \mathrm{L}$, and potassium was 3.2 $\mathrm{mEq} / \mathrm{L}$; so the IV fluid changed to dextrose $5 \%$ and normal saline with $40 \mathrm{meq} / \mathrm{L} \mathrm{KCl} \mathrm{15 \% .} \mathrm{The} \mathrm{first} \mathrm{arterial} \mathrm{blood}$ 
gas (ABG) showed metabolic acidosis. Nebulization with a 5\% saline solution and Budezonide was administrated. Serum sodium and potassium levels were measured every 6 hours, and $\mathrm{O}_{2}$ therapy was continued. One day after the admission, he had moderate dehydration, and breast milk was started. Serial sodium levels were continuously high, and changes in volume and amount of IV fluid's sodium had no effect on it. After 24 hours, he had poor response hypernatremia, moderate dehydration, and $\mathrm{O}_{2}$ dependency.

Considering the outbreak of the novel coronavirus in our country, we took an oropharyngeal swap sample and a chest CT-scan. A blood test was done for lactate dehydrogenase (LDH) and creatine phosphokinase (CPK), which were high (1320 and 371, respectively). His test result was negative for coronavirus, but his chest CT-scan showed consolidation in the upper lobe and inferior lobe's posterior segments of the right lung and ground-glass opacity (GGO) in the left lung's inferior lobe lateral base and posterior base (Figure 1). Thereafter, we started oral azithromycin and hydroxychloroquine and admitted him to the isolated COVID-19 ward. Intravenous ampicillin was discontinued, but we continued cefotaxime along with fluid therapy.

On day 3 of the admission, his situation improved, and the hemodynamic status had been stabled. The fever was resolved, and he had mild dehydration. Serial sodium and potassium check were discontinued after two consequent normal tests. $\mathrm{His} \mathrm{O}_{2}$ saturation was above $95 \%$, and there was no need for $\mathrm{O}_{2}$ therapy anymore. Unfortunately, his father discharged him despite the specialist opinion. He was stable in further telephone follow-ups.

\section{Discussion}

Pediatric case reports in COVID-19 are limited, and it seems that the disease has a milder course compared to the adult patients, but the range of the clinical features are wide (3). Most of the cases are reported in family clusters, and there is a strong correlation between the disease in family members and the child. The incidence of COVID-19 has been reported by $18.1 \%$ in children under 1 year old (2). We here presented a 2-months old boy with fever and gastrointestinal symptoms and a suspected history of contact, as his mother showed symptoms suspected to COVID-19.

Fever is the most common sign in children like our patient, but vomiting and diarrhea are very rare (3-5). Our case presented with fever and severe dehydration as well.

The oropharyngeal swap test results were negative in our case. As it has been reported to be less positive in pediatrics, and it should be kept in mind among the differential diagnosis with all aspects of the patient (2). Although the positive PCR test is considered the gold standard test for the diagnosis of COVID-19, false-negative patients are the source of the infection (3) and repeating the test increases the accuracy, which was not done in our patient due to the shortage of diagnosis facilities in the area (Northeast of Iran).

Other studies in pediatrics showed normal laboratory tests $(4,5)$, but our patient had leukocytosis, a positive CRP and high levels of LDH and CPK that can be seen in any viral infection. So, further specific diagnostic methods were needed.

In this case, the base of our clinical diagnosis was the CT scan's remarkable pattern, despite the normal chest $\mathrm{X}$-ray (CXR). As reported in other studies, the radiologic changes in the lungs can be seen in the first CXR, but a normal graph would not rule out the diagnosis of COVID-19. Chest CT-scan can detect the pathologic lesions better than a plain X-ray, and ground-glass opacity (32.7\% in one study) and segmental consolidation in bilateral lungs have been reported in pediatric patients $(2,4)$ that was seen in our patient as well.

Our patient had hypernatremia due to severe dehydration, and the response to the initial fluid resuscitation was not quite satisfactory. However, after starting oral azithromycin and hydroxychloroquine (based on the management protocol), he got better and, finally, discharged in a good health situation.

\section{Acknowledgments}

Authors tend to thank the nursing service of the Jajarm's Hospital, Northeast of Iran, for their sacrifices in the COVID-19 era.

\section{Footnotes}

Authors' Contribution: Mahsa Besharat did study concept and design, acquisition of data, drafting the manuscript, critical revision of the manuscript for important intellectual content, statistical analysis, and study supervision. Pooya Bahari Khorram did acquisition of data, Analysis, and interpretation of data, drafting the manuscript, critical revision of the manuscript for important intellectual content, and statistical analysis.

Conflict of Interests: The authors declare no conflict of interest.

Ethical Approval: The ethical approval code was SIR.GOUMS.REC.1399.061. 

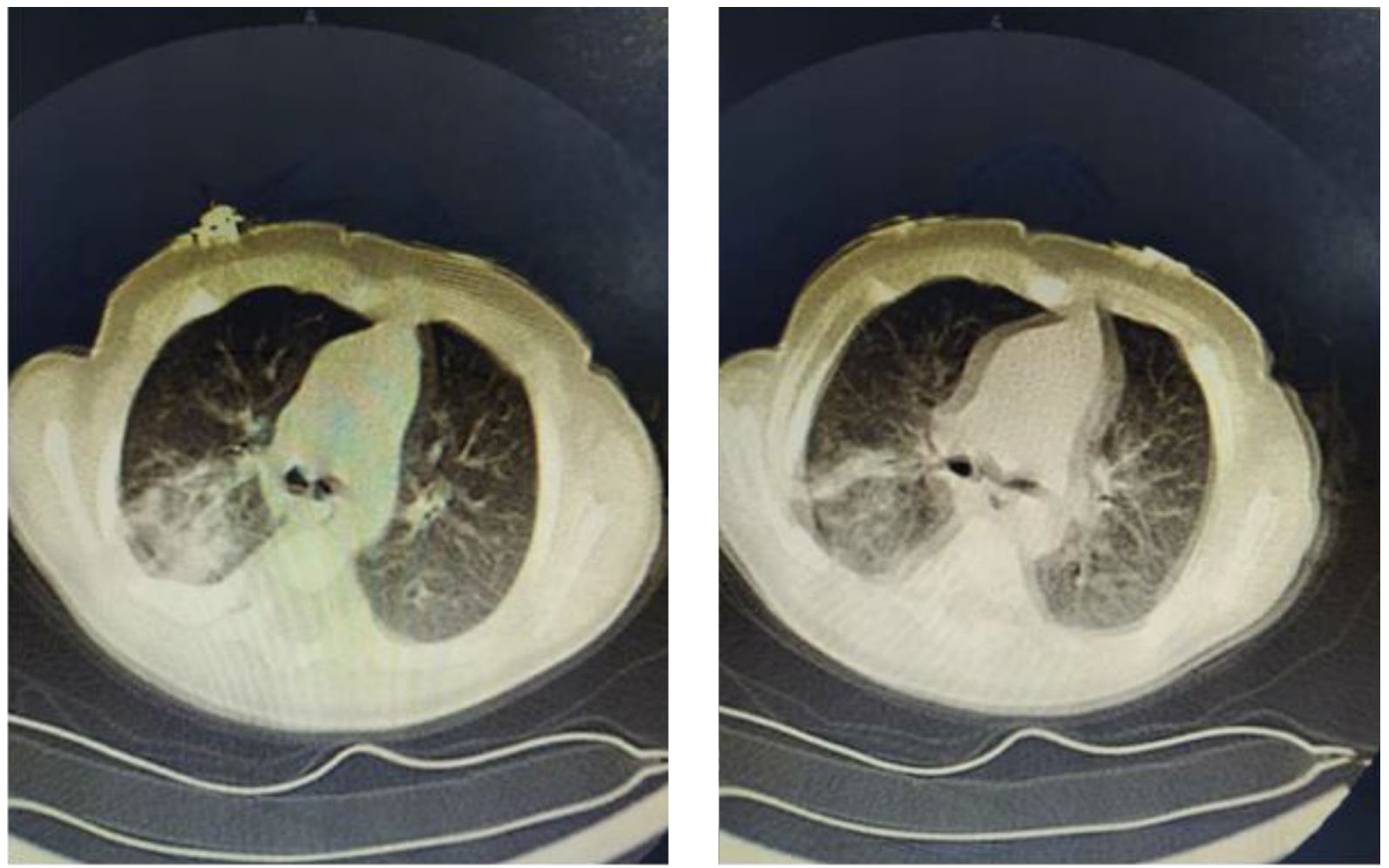

Figure 1. The chest CT-scan of the patient is shown

Funding/Support: The authors received no funding or support regarding the publication of this article.

Informed Consent: We obtained informed consent from our patient's parents.

\section{References}

1. Esper F, Shapiro ED, Weibel C, Ferguson D, Landry ML, Kahn JS. Association between a novel human coronavirus and Kawasaki disease. J Infect Dis. 2005;191(4):499-502. doi: 10.1086/428291. [PubMed: 15655771]. [PubMed Central: PMC7199489].
2. Tezer H, Bedir Demirdag T. Novel coronavirus disease (COVID-19) in children. Turk J Med Sci. 2020;50(SI-1):592-603. doi: 10.3906/sag-2004174. [PubMed: 32304191]. [PubMed Central: PMC7195991].

3. Yang P, Liu P, Li D, Zhao D. Corona virus disease 2019, a growing threat to children? J Infect. 2020;80(6):671-93. doi: 10.1016/j.jinf.2020.02.024. [PubMed: 32142929]. [PubMed Central: PMC7125808].

4. Su L, Ma X, Yu H, Zhang Z, Bian P, Han Y, et al. The different clinical characteristics of corona virus disease cases between children and their families in China - the character of children with COVID-19. Emerg Microbes Infect. 2020;9(1):707-13. doi: 10.1080/22221751.2020.1744483. [PubMed: 32208917]. [PubMed Central: PMC7103724].

5. Chen J, Zhang ZZ, Chen YK, Long QX, Tian WG, Deng HJ, et al. The clinical and immunological features of pediatric COVID-19 patients in China. Genes Dis. 2020. doi: 10.1016/j.gendis.2020.03.008. [PubMed: 32363222]. [PubMed Central: PMC7194810]. 